\title{
The Catalonian Conflict: an Interpretive Structural Model
}

\author{
Félix Martínez de la Rosa \\ Department of Mathematics \\ Universidad de Cádiz | UCA \\ Cádiz, Spain
}

\author{
Francisco Parra-Luna \\ Catedrático de Sociología jubilado \\ Miembro de la IASCYS (International \\ Academy for Systems and Cybernetic \\ Sciences) \\ Madrid, Spain
}

\author{
Rafael Pla-Lopez \\ profesor Titular de Universidad jubilado \\ de Matemática Aplicada \\ Universitat de València \\ València, Spain
}

\begin{abstract}
The methodology ISM (Interpretive Structural Modeling) is used to study the conflict in Catalonia, listing a list of factors and the influences between them, building from them the Matrix of Influences and the Matrix of Scope, from which they classify the factors into levels and a flow diagram is drawn up, concluding what the possible paths of evolution of said conflict would be.
\end{abstract}

\section{Keywords - Interpretive; structural; Constitutional recognition; political pardon; institutional normalization}

\section{Introducción}

The conflict in Catalonia has a special complexity, given the interaction of a large number of factors. In view of the existing systems approach (Forrester Systems Dynamics, "Soft Systems" by P. Checkland, Viable Systems of S. Beer, Second Order Cybernetics by H. von Foerster and others), we decided to use the methodology known as ISM (Interpretive Structural Modeling) by Warfield (1974) to try to clarify between the farrago of factors at stake, identify and enumerate them, advance hypotheses about their mutual influences and finish building the so-called Matrix of Influences and the Outcome Matrix to classify the factors in Levels and prepare the corresponding flow diagram that is supposed to clarify. All this, at least in our intention, to indicate which would be the possible ways of evolution of the Catalan conflict.

\section{List of Factors and Influences}

In the following, " $\rightarrow$ " means "influences" the factors indicated below.

a. Extension of the desire for independence in Catalonia $\rightarrow \mathrm{h}, \mathrm{i}, \mathrm{k}, \mathrm{m}, \mathrm{q}, \mathrm{ac}, \mathrm{ad}, \mathrm{ae}, \mathrm{ag}$

b. Extension of the desire for non-independence within Catalonia $\rightarrow \mathrm{c}, \mathrm{n}, \mathrm{o}, \mathrm{p}, \mathrm{v}, \mathrm{ad}$, af

c. Opposition to the independence of Catalonia in the rest of Spain $\rightarrow$ d, e, j, n, r, ad, af

d. Action of the Constitutional Court $\rightarrow \mathrm{q}, \mathrm{r}, \mathrm{u}, \mathrm{x}, \mathrm{y}, \mathrm{z}$, ae, af, ag

e. Action of the Courts of Justice $\rightarrow q, r, u, x, y, z$, ae f. Action of the Office of the Prosecutor $\rightarrow e, v, w, x, y$, $\mathrm{Z}$

g. Text of the Spanish Constitution $\rightarrow \mathrm{a}, \mathrm{c}, \mathrm{d}, \mathrm{e}, \mathrm{h}, \mathrm{o}, \mathrm{t}$, ad, af

h. Actions to change the Constitution

i. Laws approved by the Parliament of Catalonia $\rightarrow \mathrm{j}, \mathrm{o}$, ac, ae

j. Resources presented by the Government of Spain $\rightarrow d$, $\mathrm{x}$, ah

$k$. Declarations of independence of the Parliament of Catalonia $\rightarrow \mathrm{d}, \mathrm{e}, \mathrm{f}, \mathrm{j}, \mathrm{ac}$, ad

1. Laws approved by the Spanish Parliament

$\mathrm{m}$. Disobedience of laws passed by the Spanish

Parliament $\rightarrow$ e, $\mathrm{z}$

n. Declarations of the Spanish Parliament in favor of the unity of Spain $\rightarrow$ ae

or. Demonstrations in Catalonia in favor of its maintenance in the Spanish State $\rightarrow$ ad

p. Claiming the right to decide Tabarnia $\rightarrow$ ae

q. Non-compliance with laws or judicial resolutions in Catalonia $\rightarrow \mathrm{e}, \mathrm{y}, \mathrm{z}$

r. Denial of the existence of political prisoners $\rightarrow t$

s. Affirmation of the existence of political prisoners $\rightarrow$ $\mathrm{u}, \mathrm{x}$

t. Affirmation that Spain is a State of Law $\rightarrow b, r$

$u$. Denial of Spain being a State of Law $\rightarrow a, s$

v. Accusation to the pro-independence supporters of having carried out a coup d'état $\rightarrow \mathrm{z}$

w. Denial that the separatists have carried out a coup d'etat $\rightarrow$ aa

$\mathrm{x}$. Independence demonstrations $\rightarrow \mathrm{k}, \mathrm{o}$, and 
y. Police charges $\rightarrow$ a

z. Imprisonment of independentistas $\rightarrow \mathrm{a}, \mathrm{u}$

aa. Liberation of imprisoned independentistas $\rightarrow b, t$

ab.Diálogo between the Government of Spain and the

Government of Catalonia $\rightarrow b$, aa

ac.Convocation of popular consultations $\rightarrow e, y, z$, an

ad.Intervention of the State in the institutions of

Catalonia $\rightarrow \mathrm{a}, \mathrm{x}$

ae.Reclaiming the right of self-determination $\rightarrow h, p$, ac af.Rejection of the right of self-determination $\rightarrow a, x$

ag.Result of the elections to the Parliament of Catalonia

$\rightarrow \mathrm{k}, \mathrm{ab}$

ah.Result of the motion of censure against the

Government of Spain $\rightarrow$ aa, ab,

ai.Resolutions of European Courts against judicial

resolutions in Spain $\rightarrow u$

aj.Lack of support from the 28 EU governments $\rightarrow$ af

ak.Insolidarity Catalan compared to the rest of Spain $\rightarrow$

a, ae

al.Solidarity Catalan compared to the rest of Spain $\rightarrow b$, af, ah

am.Lack of proportionality of electoral systems in Spain

$\rightarrow 1$, ag

an.Prohibition of the referendum on October $1 \rightarrow \mathrm{y}, \mathrm{z}$

\section{Matrix of Influences}

The direct influence that one factor has on the others will be expressed through the so-called Matrix of Influences. The headers of the columns are the factors, and those of the rows are also. For example, if the factor a directly influences others (according to the previous list), we will put a 1 in the column of a, in the row of each factor that it influences. In those that do not influence, a 0 is set. Only the values 0 and 1 are used. In other works (for example in (Ballesteros, Ballesteros, 2008) the influence is assessed 1 (weak influence), 2 (medium influence), 3 (strong influence), or P (potential influence)), but in our view this would introduce a certain subjectivity. To avoid it, only the values 0 and 1 have been chosen.

\begin{tabular}{|c|c|c|c|c|c|c|c|c|c|c|c|c|c|c|c|c|c|c|c|c|c|c|c|c|c|c|c|c|c|c|c|c|c|c|c|c|c|c|}
\hline & $a b$ & $\mathrm{~b} c$ & $\mathrm{c}$ & & & & & & & & & & & & & & & 1 & 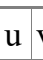 & & W & & $\mathrm{y} z$ & & & $\mathrm{ab}$ & ac & ad & ae & af & ag & ah & & aj & $\mathrm{ak}$ & al & am & an \\
\hline $\mathrm{a}$ & 0 & 00 & \begin{tabular}{l|l}
0 & 0
\end{tabular} & & 0 & 1 & 0 & 0 & 0 & 00 & 0 & 0 & 0 & & & & 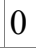 & 0 & 1 & 0 & 0 & & 11 & & 0 & 0 & 0 & 1 & 0 & 1 & 0 & 0 & 0 & 0 & 1 & 0 & 0 & 0 \\
\hline b & 0 & $\begin{array}{lll}0 & 0\end{array}$ & $\begin{array}{lll}0 & 0\end{array}$ & ) & & 0 & 0 & & & & & 0 & 00 & & & & 0 & 1 & ( & & 0 & & 00 & & 1 & 1 & 0 & 0 & 0 & 0 & 0 & 0 & 0 & 0 & 0 & 1 & 0 & 0 \\
\hline $\mathrm{c}$ & 0 & 0 & \begin{tabular}{l|l}
0 & 0 \\
\end{tabular} & & 0 & 1 & 0 & & 0 & 0 & 0 & 0 & 0 & & 0 & & 0 & 0 & 0 & 0 & 0 & 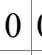 & 00 & & 0 & 0 & 0 & 0 & 0 & 0 & 0 & 0 & 0 & 0 & 0 & 0 & 0 & 0 \\
\hline $\mathrm{d}$ & 0 & \begin{tabular}{l|l}
0 & 1
\end{tabular} & 10 & ) & & 1 & 0 & & & 1 & 0 & 0 & 00 & & 0 & & 0 & 0 & 0 & 0 & 0 & 0 & 0 & & 0 & 0 & 0 & 0 & 0 & 0 & 0 & 0 & 0 & 0 & 0 & 0 & 0 & 0 \\
\hline $\mathrm{e}$ & 0 & \begin{tabular}{l|l}
0 & 1
\end{tabular} & $\begin{array}{lll}1 & 0\end{array}$ & 0 & & 1 & 0 & 0 & 0 & 10 & 0 & 1 & 0 & & & & 0 & 0 & 0 & & 0 & ( & 0 & & 0 & 0 & 1 & 0 & 0 & 0 & 0 & 0 & 0 & 0 & 0 & 0 & 0 & 0 \\
\hline $\mathrm{f}$ & 0 & 00 & $\begin{array}{ll}0 & 0\end{array}$ & ) & 0 & 0 & 0 & 0 & 0 & 1 & 0 & 0 & 0 & & 0 & & 0 & 0 & 0 & 0 & 0 & 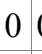 & 0 & & 0 & 0 & 0 & 0 & 0 & 0 & 0 & 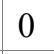 & 0 & 0 & 0 & 0 & 0 & 0 \\
\hline $\mathrm{g}$ & 0 & 00 & $\begin{array}{lll}0 & 0\end{array}$ & 0 & 0 & 0 & 0 & & 0 & 0 & 0 & 0 & 00 & & 0 & & 0 & 0 & 0 & 0 & 0 & 0 & 00 & & 0 & 0 & 0 & 0 & 0 & 0 & 0 & 0 & 0 & 0 & 0 & 0 & 0 & 0 \\
\hline $\mathrm{h}$ & 1 & 00 & $\begin{array}{lll}0 & 0\end{array}$ & 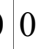 & 0 & 1 & 0 & 0 & 0 & 0 & 0 & 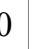 & 0 & & & & 0 & 0 & 0 & & 0 & 0 & 0 & & 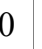 & 0 & 0 & . & 1 & 0 & 0 & 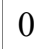 & 0 & 0 & 0 & 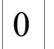 & 0 & 0 \\
\hline $\mathrm{i}$ & 1 & 00 & & 0 & 0 & 0 & 0 & 0 & 0 & 0 & 0 & 0 & 0 & ) & 0 & & 0 & 0 & 0 & 0 & 0 & 0 & 0 & & 0 & 0 & 0 & 0 & 0 & 0 & 0 & 0 & 0 & 0 & 0 & 0 & 0 & 0 \\
\hline $\mathrm{j}$ & 0 & $\begin{array}{lll}0 & 1\end{array}$ & $\begin{array}{ll}10 \\
\end{array}$ & ) & 0 & 0 & 0 & & 0 & 1 & 0 & 0 & 00 & ) & 0 & & 0 & 0 & 0 & 0 & 0 & 0 & 0 & & 0 & 0 & 0 & 0 & 0 & 0 & 0 & 0 & 0 & 0 & 0 & 0 & 0 & 0 \\
\hline $\mathrm{k}$ & 1 & 00 & $\begin{array}{lll}0 & 0\end{array}$ & 0 & 0 & 0 & 0 & . & 0 & 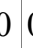 & 0 & 0 & 0 & & & & 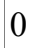 & 0 & 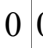 & 0 & 0 & 1 & 0 & & 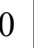 & $\sigma$ & 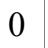 & 0 & 0 & 0 & 1 & 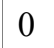 & 0 & 0 & 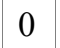 & 0 & $\sigma$ & 0 \\
\hline 1 & 0 & $\begin{array}{lll}0 & 0\end{array}$ & & ) & 0 & 0 & 0 & 0 & 0 & 0 & 0 & 0 & 0 & 0 & 0 & & 0 & 0 & 0 & 0 & 0 & 0 & 00 & & 0 & 0 & 0 & 0 & 0 & 0 & 0 & 0 & 0 & 0 & 0 & 0 & 1 & 0 \\
\hline $\mathrm{m}$ & 1 & 00 & \begin{tabular}{l|l}
0 & 0
\end{tabular} & 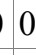 & 0 & 0 & 0 & 0 & 0 & 0 & 0 & 0 & 0 & 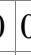 & 0 & & 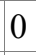 & 0 & ( & 0 & 0 & 0 & 0 & & & 0 & 0 & 0 & 0 & 0 & 0 & 0 & 0 & 0 & 0 & 0 & 0 & 0 \\
\hline $\mathrm{n}$ & 0 & $\begin{array}{lll}1 & 1\end{array}$ & $\begin{array}{lll}1 & 0\end{array}$ & ) & 0 & 0 & 0 & 0 & 0 & 0 & 0 & 0 & 0 & & & & 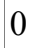 & | & 4 & 0 & 0 & 0 & 04 & & 0 & 0 & 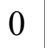 & 0 & 0 & 0 & 0 & 0 & 0 & 0 & 0 & 0 & 0 & 0 \\
\hline $\mathrm{O}$ & 0 & 10 & $\begin{array}{ll}0 & 0\end{array}$ & ) & 0 & 1 & 0 & 1 & 0 & 0 & 0 & 0 & 00 & 0 & 0 & 0 & 0 & 0 & 0 & 0 & 0 & 1 & 00 & & 0 & 0 & 0 & 0 & 0 & 0 & 0 & 0 & 0 & 0 & 0 & 0 & 0 & 0 \\
\hline $\mathrm{p}$ & 0 & 10 & $\begin{array}{ll}0 & 0\end{array}$ & ) & 0 & 0 & 0 & & 0 & 0 & 0 & 0 & 06 & & & & 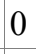 & 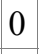 & 0 & 0 & 0 & & 0 & & & 0 & 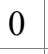 & 0 & 1 & 0 & 0 & 0 & 0 & 0 & 0 & U & 0 & 0 \\
\hline $\mathrm{q}$ & 1 & 00 & & 1 & 0 & 0 & 0 & & 0 & 0 & 0 & 0 & 0 & & & & 0 & 0 & ( & 0 & 0 & 0 & 0 & & U & 0 & 0 & 0 & 0 & 0 & 0 & U & 0 & 0 & 0 & 0 & 0 & 0 \\
\hline $\mathrm{r}$ & 0 & \begin{tabular}{l|l}
0 & 1
\end{tabular} & & 1 & 0 & 0 & . & & 0 & 0 & 0 & 0 & 0 & & 2 & & 0 & 1 & 0 & 0 & 0 & 0 & 0 & & 0 & 0 & 0 & 0 & 0 & 0 & 0 & 0 & 0 & 0 & 0 & 0 & 0 & 0 \\
\hline $\mathrm{s}$ & 0 & 00 & \begin{tabular}{l|l}
0 & 0
\end{tabular} & 0 & 0 & 0 & 0 & & 0 & 0 & 0 & U & 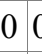 & & & & 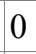 & 0 & 1 & U & 0 & 0 & 00 & & & . & 0 & 0 & 0 & 0 & 0 & 0 & 0 & 0 & U & 0 & 0 & 0 \\
\hline $\mathrm{t}$ & 0 & $\begin{array}{lll}0 & 0\end{array}$ & \begin{tabular}{l|l}
0 & 0
\end{tabular} & 0 & 0 & 1 & 0 & & 0 & 0 & 0 & 0 & 0 & & & & 0 & 0 & 0 & 0 & 0 & 0 & 0 & & 1 & 0 & 0 & 0 & 0 & 0 & 0 & 0 & 0 & 0 & 0 & 0 & 0 & 0 \\
\hline $\mathrm{u}$ & 0 & $\begin{array}{lll}0 & 0\end{array}$ & \begin{tabular}{l|l}
0 & 1 \\
\end{tabular} & 1 & 0 & 0 & 0 & & 0 & 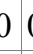 & 0 & 0 & 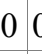 & & & & 1 & 0 & 0 & 0 & 0 & 0 & 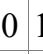 & & 0 & 0 & $\sigma$ & 0 & 0 & 0 & 0 & 0 & 1 & 0 & 0 & 0 & 0 & 0 \\
\hline $\mathrm{V}$ & 0 & 10 & \begin{tabular}{l|l}
0 & 0
\end{tabular} & 0 & 1 & 0 & 0 & & 0 & 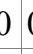 & 0 & 0 & 0 & & & & & 0 & 0 & 0 & 0 & & & & & 0 & 0 & 0 & 0 & 0 & 0 & 0 & 0 & 0 & 0 & 0 & 0 & 0 \\
\hline $\mathrm{w}$ & 0 & 00 & $\begin{array}{lll}0 & 0\end{array}$ & 0 & 1 & 0 & 0 & & 0 & 0 & 0 & 0 & 0 & 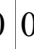 & & & 0 & 0 & 0 & 0 & 0 & 0 & 0 & & 0 & 0 & 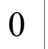 & 0 & 0 & 0 & 0 & 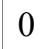 & 0 & 0 & 0 & 0 & 0 & 0 \\
\hline $\mathrm{X}$ & 0 & 00 & & 1 & 1 & 0 & 0 & & 1 & 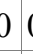 & 0 & 0 & 06 & & & & 1 & 0 & 0 & 0 & 0 & 0 & 04 & & U & 0 & 0 & 1 & 0 & 1 & 0 & 0 & 0 & 0 & 0 & 0 & 0 & 0 \\
\hline $\mathrm{y}$ & 0 & 00 & \begin{tabular}{l|l}
0 & 1 \\
\end{tabular} & 1 & 1 & 0 & 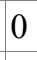 & & 0 & 0 & 0 & 0 & 0 & & & & & 4 & 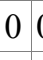 & 0 & 0 & 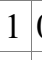 & & & & 0 & 1 & 0 & 0 & 0 & 0 & 0 & 0 & 0 & 0 & 0 & 0 & 1 \\
\hline $\mathrm{z}$ & 0 & 00 & \begin{tabular}{l|l}
0 & 1
\end{tabular} & 1 & 1 & 0 & 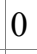 & & 0 & 0 & 0 & 1 & 0 & & & & J & 0 & & 1 & 0 & 0 & & & U & 0 & 1 & 0 & 0 & 0 & 0 & 0 & 0 & 0 & 0 & U & 0 & 1 \\
\hline aa & 0 & $\begin{array}{lll}0 & 0\end{array}$ & & & 0 & 0 & 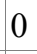 & & 0 & + & 0 & 0 & 0 & & & & & 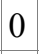 & 0 & 0 & 1 & 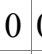 & 00 & & 0 & 1 & 0 & 0 & 0 & 0 & 0 & 1 & 0 & 0 & 0 & 0 & 0 & 0 \\
\hline$a b$ & 0 & 00 & $\begin{array}{lll}0 & 0\end{array}$ & & & 0 & 0 & & & & & 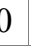 & 0 & & & & & 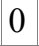 & & & 0 & & & & 0 & 0 & 0 & 0 & 0 & 0 & 1 & 1 & 0 & 0 & 0 & 0 & 0 & 0 \\
\hline
\end{tabular}




\begin{tabular}{|c|c|c|c|c|c|c|c|c|c|c|c|c|c|c|c|c|c|c|c|c|c|c|c|c|c|c|c|c|c|c|c|c|c|c|c|c|c|c|c|c|}
\hline ac & 1 & 0 & 0 & 0 & 0 & 0 & 0 & 0 & 1 & 0 & 1 & 0 & 0 & 0 & 0 & 0 & 0 & 0 & 0 & 0 & 0 & 0 & 0 & 0 & 0 & 0 & 0 & 0 & 0 & 0 & 1 & 0 & 0 & 0 & 0 & 0 & 0 & 0 & 0 & 0 \\
\hline ad & 1 & 1 & 1 & 0 & 0 & 0 & 1 & 0 & 0 & 0 & 1 & 0 & 0 & 0 & 1 & 0 & 0 & 0 & 0 & 0 & 0 & 0 & 0 & 0 & 0 & 0 & 0 & 0 & 0 & 0 & 0 & 0 & 0 & 0 & 0 & 0 & 0 & 0 & 0 & 0 \\
\hline ae & 1 & 0 & 0 & 1 & 1 & 0 & 0 & 0 & 1 & 0 & 0 & 0 & 0 & 1 & 0 & 1 & 0 & 0 & 0 & 0 & 0 & 0 & 0 & 0 & 0 & 0 & 0 & 0 & 0 & 0 & 0 & 0 & 0 & 0 & 0 & 0 & 1 & 0 & 0 & 0 \\
\hline af & 0 & 1 & 1 & 1 & 0 & 0 & 1 & 0 & 0 & 0 & 0 & 0 & 0 & 0 & 0 & 0 & 0 & 0 & 0 & 0 & 0 & 0 & 0 & 0 & 0 & 0 & 0 & 0 & 0 & 0 & 0 & 0 & 0 & 0 & 0 & 1 & 0 & 1 & 0 & 0 \\
\hline ag & 1 & 0 & 0 & 1 & 0 & 0 & 0 & 0 & 0 & 0 & 0 & 0 & 0 & 0 & 0 & 0 & 0 & 0 & 0 & 0 & 0 & 0 & 0 & 0 & 0 & 0 & 0 & 0 & 0 & 0 & 0 & 0 & 0 & 0 & 0 & 0 & 0 & 0 & 1 & 0 \\
\hline ah & 0 & 0 & 0 & 0 & 0 & 0 & 0 & 0 & 0 & 1 & 0 & 0 & 0 & 0 & 0 & 0 & 0 & 0 & 0 & 0 & 0 & 0 & 0 & 0 & 0 & 0 & 0 & 0 & 0 & 0 & 0 & 0 & 0 & 0 & 0 & 0 & 0 & 1 & 0 & 0 \\
\hline ai & 0 & 0 & 0 & 0 & 0 & 0 & 0 & 0 & 0 & 0 & 0 & 0 & 0 & 0 & 0 & 0 & 0 & 0 & 0 & 0 & 0 & 0 & 0 & 0 & 0 & 0 & 0 & 0 & 0 & 0 & 0 & 0 & 0 & 0 & 0 & 0 & 0 & 0 & 0 & 0 \\
\hline aj & 0 & 0 & 0 & 0 & 0 & 0 & 0 & 0 & 0 & 0 & 0 & 0 & 0 & 0 & 0 & 0 & 0 & 0 & 0 & 0 & 0 & 0 & 0 & 0 & 0 & 0 & 0 & 0 & 0 & 0 & 0 & 0 & 0 & 0 & 0 & 0 & 0 & 0 & 0 & 0 \\
\hline ak & 0 & 0 & 0 & 0 & 0 & 0 & 0 & 0 & 0 & 0 & 0 & 0 & 0 & 0 & 0 & 0 & 0 & 0 & 0 & 0 & 0 & 0 & 0 & 0 & 0 & 0 & 0 & 0 & 0 & 0 & 0 & 0 & 0 & 0 & 0 & 0 & 0 & 0 & 0 & 0 \\
\hline al & 0 & 0 & 0 & 0 & 0 & 0 & 0 & 0 & 0 & 0 & 0 & 0 & 0 & 0 & 0 & 0 & 0 & 0 & 0 & 0 & 0 & 0 & 0 & 0 & 0 & 0 & 0 & 0 & 0 & 0 & 0 & 0 & 0 & 0 & 0 & 0 & 0 & 0 & 0 & 0 \\
\hline am & 0 & 0 & 0 & 0 & 0 & 0 & 0 & 0 & 0 & 0 & 0 & 0 & 0 & 0 & 0 & 0 & 0 & 0 & 0 & 0 & 0 & 0 & 0 & 0 & 0 & 0 & 0 & 0 & 0 & 0 & 0 & 0 & 0 & 0 & 0 & 0 & 0 & 0 & 0 & 0 \\
\hline an & 0 & 0 & 0 & 0 & 0 & 0 & 0 & 0 & 0 & 0 & 0 & 0 & 0 & 0 & 0 & 0 & 0 & 0 & 0 & 0 & 0 & 0 & 0 & 0 & 0 & 0 & 0 & 0 & 1 & 0 & 0 & 0 & 0 & 0 & 0 & 0 & 0 & 0 & 0 & 0 \\
\hline
\end{tabular}

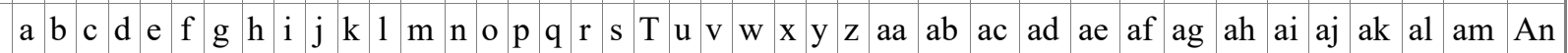

\section{Final Reach Matrix}

We assume that the factors attend to the effect of transience: It is assumed that A influences itself: in the column of $\mathrm{A}$ a 1 is placed in the row of $\mathrm{A}$. If $\mathrm{A}$ influences $\mathrm{B}$ : in the column of $\mathrm{A}$ a 1 is placed in the row of $\mathrm{B}$. If $\mathrm{A}$ influences $\mathrm{B}$, and $\mathrm{B}$ influences $\mathrm{C}$ : in the column of $\mathrm{A}$ a 1 is placed in the row of $C$. If $A$ influences $B, B$ over $C$ and $C$ over $D$ : in the column of $A$, a 1 is placed in the row of D. Applying the transience, the final scope matrix is obtained. 
Adding the numbers of each row, you get a number that indicates how many factors influence the owner of the row, which we call your power of dependence (PD).

Adding the numbers of each column, you get a number that indicates how many factors have influenced the head of the column, which we call your power of momentum (PI).
Thus, "a" would have the highest range of impulse power (34), while " $h$ " would have the highest rank of dependence power (38).

These values may be relevant in later applications of the model.

\section{Classification in Levels}


For each factor it is indicated the set of its reaches (in the upper box) and its dependencies (in the lower box) and the intersection thereof. Those factors for which the reaches coincide with the intersection will be in the first level, I.

Level I: (Scopes) $\cap$ (Dependencies) $=$ Scopes
The factors already classified are no longer considered, and those in which the remaining reaches coincide with the remaining intersection will be in the next level. And this process is repeated until all the factors are classified: \# 


\begin{tabular}{|c|c|c|c|}
\hline \multirow[t]{2}{*}{ A } & $\begin{array}{c}\text { a,b,c,d,e,f,h,i,j,k,m,n,o,p,q,r,s,t,u,v,w,x,y,z, } \\
\text { aa,ab,ac,ad,ae,af,ag,ah,an }\end{array}$ & \multirow[t]{2}{*}{$\begin{array}{c}\text { a,b,c,d,e,f,i,j,k,m,n,o,p,q,r,s,t,u,v,w,x,y,z, } \\
\text { aa,ab,ac,ad,ae,af,ag,ah,an }\end{array}$} & \multirow[t]{2}{*}{ II } \\
\hline & $\begin{array}{l}\text { a,b,c,d,e,f,i,j,k,m,n,o,p,q,r,s,t,u,v,w,x,y,z, } \\
\text { aa,ab,ac,ad,ae,af,ag,ah,ai,aj,ak,al,am,an }\end{array}$ & & \\
\hline \multirow[t]{2}{*}{ B } & $\begin{array}{c}\text { a,b,c,d,e,f,h,i,j,k,m,n,o,p,q,r,s,t,u,v,w,x,y,z, } \\
\text { aa,ab,ac,ad,ae,af,ag,ah,an }\end{array}$ & \multirow[t]{2}{*}{$\begin{array}{c}\text { a,b,c,d,e,f,i,j,k,m,n,o,p,q,r,s,t,u,v,w,x,y,z, } \\
\text { aa,ab,ac,ad,ae,af,ag,ah,an }\end{array}$} & \multirow[t]{2}{*}{ II } \\
\hline & $\begin{array}{c}\text { a,b,c,d,e,f,i,j,k,m,n,o,p,q,r,s,t,u,v,w,x,y,z, } \\
\text { aa,ab,ac,ad,ae,af,ag,ah,ai,aj,ak,al,am,an }\end{array}$ & & \\
\hline \multirow[t]{2}{*}{$\mathrm{C}$} & $\begin{array}{c}\text { a,b,c,d,e,f,h,i,j,k,m,n,o,p,q,r,s,t,u,v,w,x,y,z, } \\
\text { aa,ab,ac,ad,ae,af,ag,ah,an }\end{array}$ & \multirow[t]{2}{*}{$\begin{array}{c}\text { a,b,c,d,e,f,i,j,k,m,n,o,p,q,r,s,t,u,v,w,x,y,z, } \\
\text { aa,ab,ac,ad,ae,af,ag,ah,an }\end{array}$} & \multirow[t]{2}{*}{ II } \\
\hline & $\begin{array}{c}\text { a,b,c,d,e,f,i,j,k,m,n,o,p,q,r,s,t,u,v,w,x,y,z, } \\
\text { aa,ab,ac,ad,ae,af,ag,ah,ai,aj,ak,al,am,an }\end{array}$ & & \\
\hline \multirow[t]{2}{*}{$\mathrm{D}$} & $\begin{array}{c}\text { a,b,c,d,e,f,h,i,j,k,m,n,o,p,q,r,s,t,u,v,w,x,y,z, } \\
\text { aa,ab,ac,ad,ae,af,ag,ah,an }\end{array}$ & \multirow[t]{2}{*}{$\begin{array}{c}\text { a,b,c,d,e,f,i,j,k,m,n,o,p,q,r,s,t,u,v,w,x,y,z, } \\
\text { aa,ab,ac,ad,ae,af,ag,ah,an }\end{array}$} & \multirow[t]{2}{*}{ II } \\
\hline & $\begin{array}{c}\text { a,b,c,d,e,f,i,j,k,m,n,o,p,q,r,s,t,u,v,w,x,y,z, } \\
\text { aa,ab,ac,ad,ae,af,ag,ah,ai,aj,ak,al,am,an }\end{array}$ & & \\
\hline \multirow[t]{2}{*}{$\mathrm{E}$} & $\begin{array}{c}\text { a,b,c,d,e,f,h,i,j,k,m,n,o,p,q,r,s,t,u,v,w,x,y,z, } \\
\text { aa,ab,ac,ad,ae,af,ag,ah,an }\end{array}$ & \multirow[t]{2}{*}{$\begin{array}{c}\text { a,b,c,d,e,f,i,j,k,m,n,o,p,q,r,s,t,u,v,w,x,y,z, } \\
\text { aa,ab,ac,ad,ae,af,ag,ah,an }\end{array}$} & \multirow[t]{2}{*}{ II } \\
\hline & $\begin{array}{l}\text { a,b,c,d,e,f,i,j,k,m,n,o,p,q,r,s,t,u,v,w,x,y,z, } \\
\text { aa,ab,ac,ad,ae,af,ag,ah,ai,aj,ak,al,am,an }\end{array}$ & & \\
\hline \multirow[t]{2}{*}{$\mathrm{F}$} & $\begin{array}{c}\text { a,b,c,d,e,f,h,i,j,k,m,n,o,p,q,r,s,t,u,v,w,x,y,z, } \\
\text { aa,ab,ac,ad,ae,af,ag,ah,an }\end{array}$ & \multirow[t]{2}{*}{$\begin{array}{c}\text { a,b,c,d,e,f,i,j,k,m,n,o,p,q,r,s,t,u,v,w,x,y,z, } \\
\text { aa,ab,ac,ad,ae,af,ag,ah,an }\end{array}$} & \multirow[t]{2}{*}{ II } \\
\hline & $\begin{array}{l}\text { a,b,c,d,e,f,i,j,k,m,n,o,p,q,r,s,t,u,v,w,x,y,z, } \\
\text { aa,ab,ac,ad,ae,af,ag,ah,ai,aj,ak,al,am,an }\end{array}$ & & \\
\hline \multirow[t]{2}{*}{$\mathrm{G}$} & $\begin{array}{c}\text { a,b,c,d,e,f,g,h,i,j,k,m,n,o,p,q,r,s,t,u,v,w,x,y,z, } \\
\text { aa,ab,ac,ad,ae,af,ag,ah,an }\end{array}$ & \multirow[t]{2}{*}{$\mathrm{g}$} & \multirow[t]{2}{*}{ III } \\
\hline & $\mathrm{g}$ & & \\
\hline \multirow[t]{2}{*}{$\mathrm{H}$} & $\mathrm{h}$ & \multirow[t]{2}{*}{$\mathrm{h}$} & \multirow[t]{2}{*}{ I } \\
\hline & $\begin{array}{c}\text { a,b,c,d,e,f,h,i,j,k,m,n,o,p,q,r,s,t,u,v,w,x,y,z, } \\
\text { aa,ab,ac,ad,ae,af,ag,ah,ai,aj,ak,al,am,an }\end{array}$ & & \\
\hline \multirow[t]{2}{*}{ I } & $\begin{array}{c}\text { a,b,c,d,e,f,h,i,j,k,m,n,o,p,q,r,s,t,u,v,w,x,y,z, } \\
\text { aa,ab,ac,ad,ae,af,ag,ah,an }\end{array}$ & \multirow[t]{2}{*}{$\begin{array}{c}\text { a,b,c,d,e,f,i,j,k,m,n,o,p,q,r,s,t,u,v,w,x,y,z, } \\
\text { aa,ab,ac,ad,ae,af,ag,ah,an }\end{array}$} & \multirow[t]{2}{*}{ II } \\
\hline & $\begin{array}{c}\text { a,b,c,d,e,f,i,j,k,m,n,o,p,q,r,s,t,u,v,w,x,y,z, } \\
\text { aa,ab,ac,ad,ae,af,ag,ah,ai,aj,ak,al,am,an }\end{array}$ & & \\
\hline \multirow[t]{2}{*}{$\mathrm{J}$} & $\begin{array}{c}\text { a,b,c,d,e,f,h,i,j,k,m,n,o,p,q,r,s,t,u,v,w,x,y,z, } \\
\text { aa,ab,ac,ad,ae,af,ag,ah,an }\end{array}$ & \multirow[t]{2}{*}{$\begin{array}{c}\text { a,b,c,d,e,f,i,j,k,m,n,o,p,q,r,s,t,u,v,w,x,y,z, } \\
\text { aa,ab,ac,ad,ae,af,ag,ah,an }\end{array}$} & \multirow[t]{2}{*}{ II } \\
\hline & $\begin{array}{l}\text { a,b,c,d,e,f,i,j,k,m,n,o,p,q,r,s,t,u,v,w,x,y,z, } \\
\text { aa,ab,ac,ad,ae,af,ag,ah,ai,aj,ak,al,am,an }\end{array}$ & & \\
\hline \multirow[t]{2}{*}{$\mathrm{K}$} & $\begin{array}{c}\text { a,b,c,d,e,f,h,i,j,k,m,n,o,p,q,r,s,t,u,v,w,x,y,z, } \\
\text { aa,ab,ac,ad,ae,af,ag,ah,an }\end{array}$ & \multirow[t]{2}{*}{$\begin{array}{c}\text { a,b,c,d,e,f,i,j,k,m,n,o,p,q,r,s,t,u,v,w,x,y,z, } \\
\text { aa,ab,ac,ad,ae,af,ag,ah,an }\end{array}$} & \multirow[t]{2}{*}{ II } \\
\hline & $\begin{array}{l}\text { a,b,c,d,e,f,i,j,k,m,n,o,p,q,r,s,t,u,v,w,x,y,z, } \\
\text { aa,ab,ac,ad,ae,af,ag,ah,ai,aj,ak,al,am,an }\end{array}$ & & \\
\hline \multirow[t]{2}{*}{$\mathrm{L}$} & 1 & \multirow[t]{2}{*}{1} & I \\
\hline & $1, \mathrm{am}$ & & \\
\hline
\end{tabular}




\begin{tabular}{|c|c|c|c|}
\hline \multirow[t]{2}{*}{$\mathrm{m}$} & $\begin{array}{c}\text { a,b,c,d,e,f,h,i,j,k,m,n,o,p,q,r,s,t,u,v,w,x,y,z, } \\
\text { aa,ab,ac,ad,ae,af,ag,ah,an }\end{array}$ & \multirow[t]{2}{*}{$\begin{array}{c}\text { a,b,c,d,e,f,i,j,k,m,n,o,p,q,r,s,t,u,v,w,x,y,z, } \\
\text { aa,ab,ac,ad,ae,af,ag,ah,an }\end{array}$} & \multirow[t]{2}{*}{ II } \\
\hline & $\begin{array}{l}\text { a,b,c,d,e,f,i,j,k,m,n,o,p,q,r,s,t,u,v,w,x,y,z, } \\
\text { aa,ab,ac,ad,ae,af,ag,ah,ai,aj,ak,al,am,an }\end{array}$ & & \\
\hline \multirow[t]{2}{*}{$\mathrm{n}$} & $\begin{array}{c}\text { a,b,c,d,e,f,h,i,j,k,m,n,o,p,q,r,s,t,u,v,w,x,y,z, } \\
\text { aa,ab,ac,ad,ae,af,ag,ah,an }\end{array}$ & \multirow[t]{2}{*}{$\begin{array}{c}\text { a,b,c,d,e,f,i,j,k,m,n,o,p,q,r,s,t,u,v,w,x,y,z, } \\
\text { aa,ab,ac,ad,ae,af,ag,ah,an }\end{array}$} & \multirow[t]{2}{*}{ II } \\
\hline & $\begin{array}{c}\text { a,b,c,d,e,f,i,j,k,m,n,o,p,q,r,s,t,u,v,w,x,y,z, } \\
\text { aa,ab,ac,ad,ae,af,ag,ah,ai,aj,ak,al,am,an }\end{array}$ & & \\
\hline \multirow[t]{2}{*}{ o } & $\begin{array}{c}\text { a,b,c,d,e,f,h,i,j,k,m,n,o,p,q,r,s,t,u,v,w,x,y,z, } \\
\text { aa,ab,ac,ad,ae,af,ag,ah,an }\end{array}$ & \multirow[t]{2}{*}{$\begin{array}{c}\text { a,b,c,d,e,f,i,j,k,m,n,o,p,q,r,s,t,u,v,w,x,y,z, } \\
\text { aa,ab,ac,ad,ae,af,ag,ah,an }\end{array}$} & \multirow[t]{2}{*}{ II } \\
\hline & $\begin{array}{l}\text { a,b,c,d,e,f,i,j,k,m,n,o,p,q,r,s,t,u,v,w,x,y,z, } \\
\text { aa,ab,ac,ad,ae,af,ag,ah,ai,aj,ak,al,am,an }\end{array}$ & & \\
\hline \multirow[t]{2}{*}{$\mathrm{p}$} & $\begin{array}{c}\text { a,b,c,d,e,f,h,i,j,k,m,n,o,p,q,r,s,t,u,v,w,x,y,z, } \\
\text { aa,ab,ac,ad,ae,af,ag,ah,an }\end{array}$ & \multirow[t]{2}{*}{$\begin{array}{c}\text { a,b,c,d,e,f,i,j,k,m,n,o,p,q,r,s,t,u,v,w,x,y,z, } \\
\text { aa,ab,ac,ad,ae,af,ag,ah,an }\end{array}$} & \multirow[t]{2}{*}{ II } \\
\hline & $\begin{array}{c}\text { a,b,c,d,e,f,i,j,k,m,n,o,p,q,r,s,t,u,v,w,x,y,z, } \\
\text { aa,ab,ac,ad,ae,af,ag,ah,ai,aj,ak,al,am,an }\end{array}$ & & \\
\hline \multirow[t]{2}{*}{$\mathrm{q}$} & $\begin{array}{c}\text { a,b,c,d,e,f,h,i,j,k,m,n,o,p,q,r,s,t,u,v,w,x,y,z, } \\
\text { aa,ab,ac,ad,ae,af,ag,ah,an }\end{array}$ & \multirow[t]{2}{*}{$\begin{array}{c}\text { a,b,c,d,e,f,i,j,k,m,n,o,p,q,r,s,t,u,v,w,x,y,z, } \\
\text { aa,ab,ac,ad,ae,af,ag,ah,an }\end{array}$} & \multirow[t]{2}{*}{ II } \\
\hline & $\begin{array}{l}\text { a,b,c,d,e,f,i,j,k,m,n,o,p,q,r,s,t,u,v,w,x,y,z, } \\
\text { aa,ab,ac,ad,ae,af,ag,ah,ai,aj,ak,al,am,an }\end{array}$ & & \\
\hline \multirow[t]{2}{*}{$\mathrm{r}$} & $\begin{array}{c}\text { a,b,c,d,e,f,h,i,j,k,m,n,o,p,q,r,s,t,u,v,w,x,y,z, } \\
\text { aa,ab,ac,ad,ae,af,ag,ah,an }\end{array}$ & \multirow[t]{2}{*}{$\begin{array}{c}\text { a,b,c,d,e,f,i,j,k,m,n,o,p,q,r,s,t,u,v,w,x,y,z, } \\
\text { aa,ab,ac,ad,ae,af,ag,ah,an }\end{array}$} & \multirow[t]{2}{*}{ II } \\
\hline & $\begin{array}{l}\text { a,b,c,d,e,f,i,j,k,m,n,o,p,q,r,s,t,u,v,w,x,y,z, } \\
\text { aa,ab,ac,ad,ae,af,ag,ah,ai,aj,ak,al,am,an }\end{array}$ & & \\
\hline \multirow[t]{2}{*}{$\mathrm{s}$} & $\begin{array}{c}\text { a,b,c,d,e,f,h,i,j,k,m,n,o,p,q,r,s,t,u,v,w,x,y,z, } \\
\text { aa,ab,ac,ad,ae,af,ag,ah,an }\end{array}$ & \multirow[t]{2}{*}{$\begin{array}{c}\text { a,b,c,d,e,f,i,j,k,m,n,o,p,q,r,s,t,u,v,w,x,y,z, } \\
\text { aa,ab,ac,ad,ae,af,ag,ah,an }\end{array}$} & \multirow[t]{2}{*}{ II } \\
\hline & $\begin{array}{l}\text { a,b,c,d,e,f,i,j,k,m,n,o,p,q,r,s,t,u,v,w,x,y,z, } \\
\text { aa,ab,ac,ad,ae,af,ag,ah,ai,aj,ak,al,am,an }\end{array}$ & & \\
\hline \multirow[t]{2}{*}{$\mathrm{t}$} & $\begin{array}{c}\text { a,b,c,d,e,f,h,i,j,k,m,n,o,p,q,r,s,t,u,v,w,x,y,z, } \\
\text { aa,ab,ac,ad,ae,af,ag,ah,an }\end{array}$ & \multirow[t]{2}{*}{$\begin{array}{c}\text { a,b,c,d,e,f,i,j,k,m,n,o,p,q,r,s,t,u,v,w,x,y,z, } \\
\text { aa,ab,ac,ad,ae,af,ag,ah,an }\end{array}$} & \multirow[t]{2}{*}{ II } \\
\hline & $\begin{array}{l}\text { a,b,c,d,e,f,i,j,k,m,n,o,p,q,r,s,t,u,v,w,x,y,z, } \\
\text { aa,ab,ac,ad,ae,af,ag,ah,ai,aj,ak,al,am,an }\end{array}$ & & \\
\hline \multirow[t]{2}{*}{$\mathrm{u}$} & $\begin{array}{c}\text { a,b,c,d,e,f,h,i,j,k,m,n,o,p,q,r,s,t,u,v,w,x,y,z, } \\
\text { aa,ab,ac,ad,ae,af,ag,ah,an }\end{array}$ & \multirow[t]{2}{*}{$\begin{array}{c}\text { a,b,c,d,e,f,i,j,k,m,n,o,p,q,r,s,t,u,v,w,x,y,z, } \\
\text { aa,ab,ac,ad,ae,af,ag,ah,an }\end{array}$} & \multirow[t]{2}{*}{ II } \\
\hline & $\begin{array}{l}\text { a,b,c,d,e,f,i,j,k,m,n,o,p,q,r,s,t,u,v,w,x,y,z, } \\
\text { aa,ab,ac,ad,ae,af,ag,ah,ai,aj,ak,al,am,an }\end{array}$ & & \\
\hline \multirow[t]{2}{*}{$\mathrm{v}$} & $\begin{array}{c}\text { a,b,c,d,e,f,h,i,j,k,m,n,o,p,q,r,s,t,u,v,w,x,y,z, } \\
\text { aa,ab,ac,ad,ae,af,ag,ah,an }\end{array}$ & \multirow[t]{2}{*}{$\begin{array}{c}\text { a,b,c,d,e,f,i,j,k,m,n,o,p,q,r,s,t,u,v,w,x,y,z, } \\
\text { aa,ab,ac,ad,ae,af,ag,ah,an }\end{array}$} & \multirow[t]{2}{*}{ II } \\
\hline & $\begin{array}{l}\text { a,b,c,d,e,f,i,j,k,m,n,o,p,q,r,s,t,u,v,w,x,y,z, } \\
\text { aa,ab,ac,ad,ae,af,ag,ah,ai,aj,ak,al,am,an }\end{array}$ & & \\
\hline \multirow[t]{2}{*}{ w } & $\begin{array}{c}\text { a,b,c,d,e,f,h,i,j,k,m,n,o,p,q,r,s,t,u,v,w,x,y,z, } \\
\text { aa,ab,ac,ad,ae,af,ag,ah,an }\end{array}$ & \multirow[t]{2}{*}{$\begin{array}{c}\text { a,b,c,d,e,f,i,j,k,m,n,o,p,q,r,s,t,u,v,w,x,y,z, } \\
\text { aa,ab,ac,ad,ae,af,ag,ah,an }\end{array}$} & \multirow[t]{2}{*}{ II } \\
\hline & $\begin{array}{l}\text { a,b,c,d,e,f,i,j,k,m,n,o,p,q,r,s,t,u,v,w,x,y,z, } \\
\text { aa,ab,ac,ad,ae,af,ag,ah,ai,aj,ak,al,am,an }\end{array}$ & & \\
\hline \multirow[t]{2}{*}{$\mathrm{X}$} & $\begin{array}{c}\text { a,b,c,d,e,f,h,i,j,k,m,n,o,p,q,r,s,t,u,v,w,x,y,z, } \\
\text { aa,ab,ac,ad,ae,af,ag,ah,an }\end{array}$ & $\begin{array}{c}\text { a,b,c,d,e,f,i,j,k,m,n,o,p,q,r,s,t,u,v,w,x,y,z, } \\
\text { aa,ab,ac,ad,ae,af,ag,ah,an }\end{array}$ & II \\
\hline & $\begin{array}{l}\text { a,b,c,d,e,f,i,j,k,m,n,o,p,q,r,s,t,u,v,w,x,y,z, } \\
\text { aa,ab,ac,ad,ae,af,ag,ah,ai,aj,ak,al,am,an }\end{array}$ & & \\
\hline $\mathrm{y}$ & $\begin{array}{c}\text { a,b,c,d,e,f,h,i,j,k,m,n,o,p,q,r,s,t,u,v,w,x,y,z, } \\
\text { aa,ab,ac,ad,ae,af,ag,ah,an }\end{array}$ & $\begin{array}{c}\text { a,b,c,d,e,f,i,j,k,m,n,o,p,q,r,s,t,u,v,w,x,y,z, } \\
\text { aa,ab,ac,ad,ae,af,ag,ah,an }\end{array}$ & II \\
\hline & $\begin{array}{l}\text { a,b,c,d,e,f,i,j,k,m,n,o,p,q,r,s,t,u,v,w,x,y,z, } \\
\text { aa,ab,ac,ad,ae,af,ag,ah,ai,aj,ak,al,am,an }\end{array}$ & & \\
\hline $\mathrm{z}$ & $\begin{array}{c}\text { a,b,c,d,e,f,h,i,j,k,m,n,o,p,q,r,s,t,u,v,w,x,y,z, } \\
\text { aa,ab,ac,ad,ae,af,ag,ah,an }\end{array}$ & $\begin{array}{c}\text { a,b,c,d,e,f,i,j,k,m,n,o,p,q,r,s,t,u,v,w,x,y,z, } \\
\text { aa,ab,ac,ad,ae,af,ag,ah,an }\end{array}$ & II \\
\hline
\end{tabular}




\begin{tabular}{|c|c|c|c|}
\hline & $\begin{array}{c}\text { a,b,c,d,e,f,i,j,k,m,n,o,p,q,r,s,t,u,v,w,x,y,z, } \\
\text { aa,ab,ac,ad,ae,af,ag,ah,ai,aj,ak,al,am,an }\end{array}$ & & \\
\hline \multirow[t]{2}{*}{ aa } & $\begin{array}{c}\text { a,b,c,d,e,f,h,i,j,k,m,n,o,p,q,r,s,t,u,v,w,x,y,z, } \\
\text { aa,ab,ac,ad,ae,af,ag,ah,an }\end{array}$ & \multirow[t]{2}{*}{$\begin{array}{c}\text { a,b,c,d,e,f,i,j,k,m,n,o,p,q,r,s,t,u,v,w,x,y,z, } \\
\text { aa,ab,ac,ad,ae,af,ag,ah,an }\end{array}$} & \multirow[t]{2}{*}{ II } \\
\hline & $\begin{array}{c}\text { a,b,c,d,e,f,i,j,k,m,n,o,p,q,r,s,t,u,v,w,x,y,z, } \\
\text { aa,ab,ac,ad,ae,af,ag,ah,ai,aj,ak,al,am,an }\end{array}$ & & \\
\hline \multirow[t]{2}{*}{$a b$} & $\begin{array}{c}\text { a,b,c,d,e,f,h,i,j,k,m,n,o,p,q,r,s,t,u,v,w,x,y,z, } \\
\text { aa,ab,ac,ad,ae,af,ag,ah,an }\end{array}$ & \multirow[t]{2}{*}{$\begin{array}{c}\text { a,b,c,d,e,f,i,j,k,m,n,o,p,q,r,s,t,u,v,w,x,y,z, } \\
\text { aa,ab,ac,ad,ae,af,ag,ah,an }\end{array}$} & \multirow[t]{2}{*}{ II } \\
\hline & $\begin{array}{c}\text { a,b,c,d,e,f,i,j,k,m,n,o,p,q,r,s,t,u,v,w,x,y,z, } \\
\text { aa,ab,ac,ad,ae,af,ag,ah,ai,aj,ak,al,am,an }\end{array}$ & & \\
\hline \multirow[t]{2}{*}{$\mathrm{ac}$} & $\begin{array}{c}\text { a,b,c,d,e,f,h,i,j,k,m,n,o,p,q,r,s,t,u,v,w,x,y,z, } \\
\text { aa,ab,ac,ad,ae,af,ag,ah,an }\end{array}$ & \multirow[t]{2}{*}{$\begin{array}{c}\text { a,b,c,d,e,f,i,j,k,m,n,o,p,q,r,s,t,u,v,w,x,y,z, } \\
\text { aa,ab,ac,ad,ae,af,ag,ah,an }\end{array}$} & \multirow[t]{2}{*}{ II } \\
\hline & $\begin{array}{c}\text { a,b,c,d,e,f,i,j,k,m,n,o,p,q,r,s,t,u,v,w,x,y,z, } \\
\text { aa,ab,ac,ad,ae,af,ag,ah,ai,aj,ak,al,am,an }\end{array}$ & & \\
\hline \multirow[t]{2}{*}{ ad } & $\begin{array}{c}\text { a,b,c,d,e,f,h,i,j,k,m,n,o,p,q,r,s,t,u,v,w,x,y,z, } \\
\text { aa,ab,ac,ad,ae,af,ag,ah,an }\end{array}$ & \multirow[t]{2}{*}{$\begin{array}{c}\text { a,b,c,d,e,f,i,j,k,m,n,o,p,q,r,s,t,u,v,w,x,y,z, } \\
\text { aa,ab,ac,ad,ae,af,ag,ah,an }\end{array}$} & \multirow[t]{2}{*}{ II } \\
\hline & $\begin{array}{c}\text { a,b,c,d,e,f,i,j,k,m,n,o,p,q,r,s,t,u,v,w,x,y,z, } \\
\text { aa,ab,ac,ad,ae,af,ag,ah,ai,aj,ak,al,am,an }\end{array}$ & & \\
\hline \multirow[t]{2}{*}{ ae } & $\begin{array}{c}\text { a,b,c,d,e,f,h,i,j,k,m,n,o,p,q,r,s,t,u,v,w,x,y,z, } \\
\text { aa,ab,ac,ad,ae,af,ag,ah,an }\end{array}$ & \multirow[t]{2}{*}{$\begin{array}{c}\text { a,b,c,d,e,f,i,j,k,m,n,o,p,q,r,s,t,u,v,w,x,y,z, } \\
\text { aa,ab,ac,ad,ae,af,ag,ah,an }\end{array}$} & \multirow[t]{2}{*}{ II } \\
\hline & $\begin{array}{c}\text { a,b,c,d,e,f,i,j,k,m,n,o,p,q,r,s,t,u,v,w,x,y,z, } \\
\text { aa,ab,ac,ad,ae,af,ag,ah,ai,aj,ak,al,am,an }\end{array}$ & & \\
\hline \multirow[t]{2}{*}{ af } & $\begin{array}{c}\text { a,b,c,d,e,f,h,i,j,k,m,n,o,p,q,r,s,t,u,v,w,x,y,z, } \\
\text { aa,ab,ac,ad,ae,af,ag,ah,an }\end{array}$ & \multirow[t]{2}{*}{$\begin{array}{c}\text { a,b,c,d,e,f,i,j,k,m,n,o,p,q,r,s,t,u,v,w,x,y,z, } \\
\text { aa,ab,ac,ad,ae,af,ag,ah,an }\end{array}$} & \multirow[t]{2}{*}{ II } \\
\hline & $\begin{array}{c}\text { a,b,c,d,e,f,i,j,k,m,n,o,p,q,r,s,t,u,v,w,x,y,z, } \\
\text { aa,ab,ac,ad,ae,af,ag,ah,ai,aj,ak,al,am,an }\end{array}$ & & \\
\hline \multirow[t]{2}{*}{ ag } & $\begin{array}{c}\text { a,b,c,d,e,f,h,i,j,k,m,n,o,p,q,r,s,t,u,v,w,x,y,z, } \\
\text { aa,ab,ac,ad,ae,af,ag,ah,an }\end{array}$ & \multirow[t]{2}{*}{$\begin{array}{c}\text { a,b,c,d,e,f,i,j,k,m,n,o,p,q,r,s,t,u,v,w,x,y,z, } \\
\text { aa,ab,ac,ad,ae,af,ag,ah,an }\end{array}$} & \multirow[t]{2}{*}{ II } \\
\hline & $\begin{array}{c}\text { a,b,c,d,e,f,i,j,k,m,n,o,p,q,r,s,t,u,v,w,x,y,z, } \\
\text { aa,ab,ac,ad,ae,af,ag,ah,ai,aj,ak,al,am,an }\end{array}$ & & \\
\hline \multirow[t]{2}{*}{ ah } & $\begin{array}{c}\text { a,b,c,d,e,f,h,i,j,k,m,n,o,p,q,r,s,t,u,v,w,x,y,z, } \\
\text { aa,ab,ac,ad,ae,af,ag,ah,an }\end{array}$ & \multirow[t]{2}{*}{$\begin{array}{c}\text { a,b,c,d,e,f,i,j,k,m,n,o,p,q,r,s,t,u,v,w,x,y,z, } \\
\text { aa,ab,ac,ad,ae,af,ag,ah,an }\end{array}$} & \multirow[t]{2}{*}{ II } \\
\hline & $\begin{array}{c}\text { a,b,c,d,e,f,i,j,k,m,n,o,p,q,r,s,t,u,v,w,x,y,z, } \\
\text { aa,ab,ac,ad,ae,af,ag,ah,ai,aj,ak,al,am,an }\end{array}$ & & \\
\hline \multirow[t]{2}{*}{ ai } & $\begin{array}{c}\text { a,b,c,d,e,f,h,i,j,k,m,n,o,p,q,r,s,t,u,v,w,x,y,z, } \\
\text { aa,ab,ac,ad,ae,af,ag,ah,ai,an }\end{array}$ & \multirow[t]{2}{*}{ ai } & \multirow[t]{2}{*}{ III } \\
\hline & ai & & \\
\hline \multirow[t]{2}{*}{ aj } & $\begin{array}{c}\text { a,b,c,d,e,f,h,i,j,k,m,n,o,p,q,r,s,t,u,v,w,x,y,z, } \\
\text { aa,ab,ac,ad,ae,af,ag,ah,aj,an }\end{array}$ & \multirow[t]{2}{*}{ aj } & \multirow[t]{2}{*}{ III } \\
\hline & aj & & \\
\hline \multirow[t]{2}{*}{ ak } & $\begin{array}{c}\text { a,b,c,d,e,f,h,i,j,k,m,n,o,p,q,r,s,t,u,v,w,x,y,z, } \\
\text { aa,ab,ac,ad,ae,af,ag,ah,ak,an }\end{array}$ & \multirow[t]{2}{*}{$\mathrm{Ak}$} & \multirow[t]{2}{*}{ III } \\
\hline & ak & & \\
\hline al & $\begin{array}{c}\text { a,b,c,d,e,f,h,i,j,k,m,n,o,p,q,r,s,t,u,v,w,x,y,z, } \\
\text { aa,ab,ac,ad,ae,af,ag,ah,al,an }\end{array}$ & $\mathrm{Al}$ & III \\
\hline & al & & \\
\hline am & $\begin{array}{c}\text { a,b,c,d,e,f,h,i,j,k,l,m,n,o,p,q,r,s,t,u,v,w,x,y,z, } \\
\text { aa,ab,ac,ad,ae,af,ag,ah,am,an }\end{array}$ & $\mathrm{Am}$ & III \\
\hline & am & & \\
\hline an & $\begin{array}{c}\text { a,b,c,d,e,f,h,i,j,k,m,n,o,p,q,r,s,t,u,v,w,x,y,z, } \\
\text { aa,ab,ac,ad,ae,af,ag,ah,an }\end{array}$ & $\begin{array}{c}\text { a,b,c,d,e,f,i,j,k,m,n,o,p,q,r,s,t,u,v,w,x,y,z, } \\
\text { aa,ab,ac,ad,ae,af,ag,ah,an }\end{array}$ & II \\
\hline & $\begin{array}{c}\text { a,b,c,d,e,f,i,j,k,m,n,o,p,q,r,s,t,u,v,w,x,y,z, } \\
\text { aa,ab,ac,ad,ae,af,ag,ah,ai,aj,ak,al,am,an }\end{array}$ & & \\
\hline
\end{tabular}




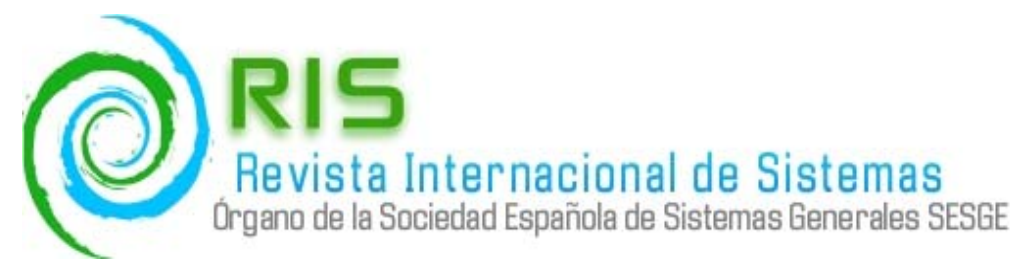

Let us indicate that the factors of a level influence, directly or indirectly, the factors of previous levels (those of III over those of II and I, those of II over those of I), which will help us to graphically represent the diagram of flows.

Spanish used in this diagram does not hinder their understanding as a whole.

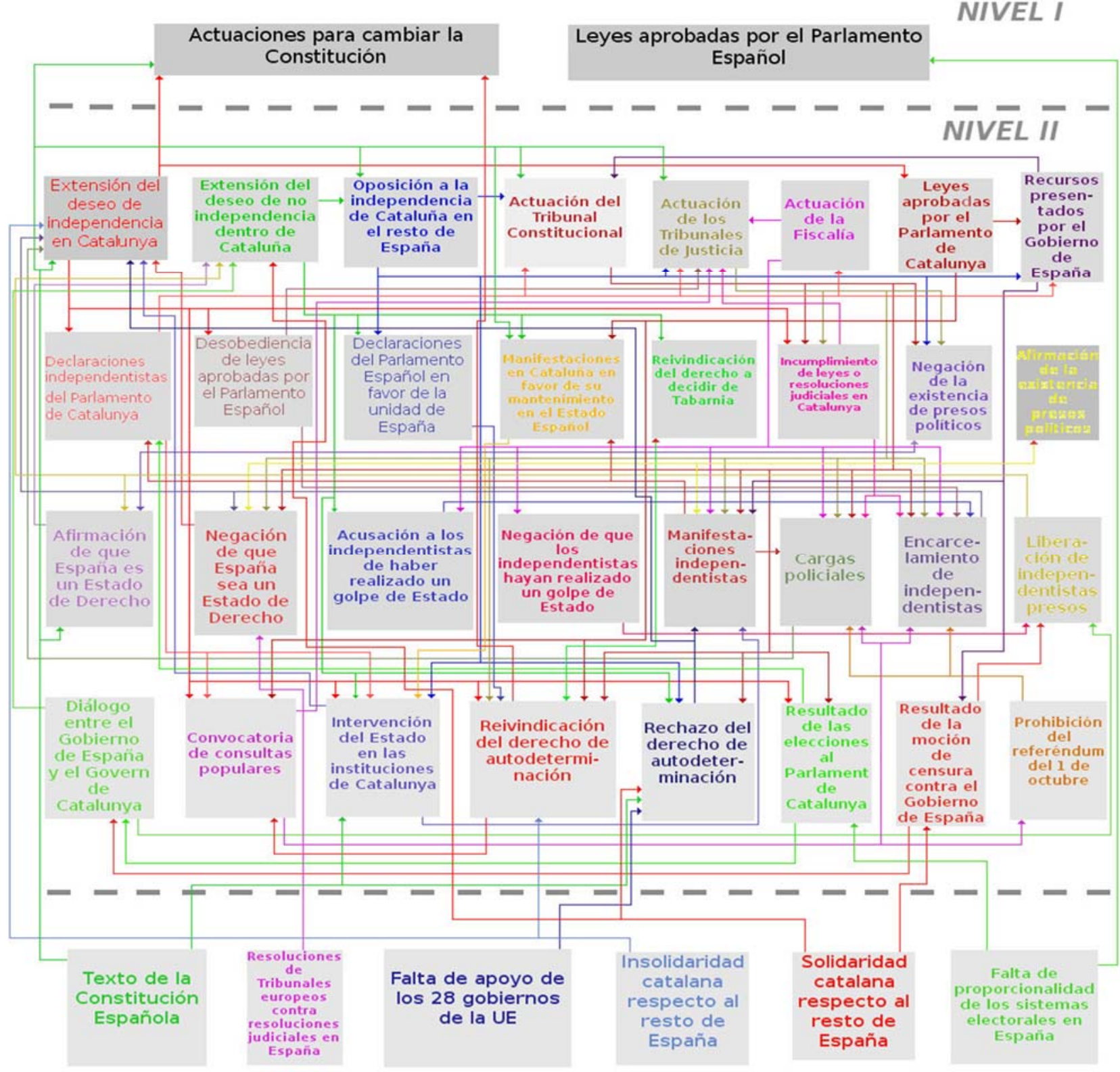

NIVEL III

FLOWCHART: For a better discernment, the factors are represented (grouped by levels) with different colors, and their direct influences by lines of the color of the origin of the influence, ending in arrowheads. If the influence is reciprocal, the other arrowhead is represented by the color of the other factor. Naturally, transitivity can be visualized by following the arrows

NOTE The 40 concepts included in this Flowchart (in Spanis $\bar{h}$ ) are already translated into the first list of "Factors and Influences" in English. It is to be expected that the 


\section{Conclusions}

In view of this structure of relations between the 40 variables used, we have started from the factors that seem to be most at the origin of the problem, verifying the significance of the following five causal paths:

1. From "Resolutions of the European Courts" $\rightarrow$ Spain is not a state of law $\rightarrow$ Extension of the desire for independence $\rightarrow$ Actions for constitutional changes

2. From "Lack of support from the 28 EU states" $\rightarrow$ Rejection of the right to self-determination $\rightarrow$ Extension of the desire for independence $\rightarrow$ Declarations of independence of the Parliament of Catalonia $\rightarrow$ Action of the courts of justice.

3. From "Text of the Spanish Constitution" $\rightarrow$ Intervention of the Central State $\rightarrow$ Extension of the desire for independence $\rightarrow$ Actions for constitutional changes

4. From "Catalan Solidarity with the rest of Spain" $\rightarrow$ Outcome of the motion of censure against the Government of Spain $\rightarrow$ Dialogue between the Government of Spain and the Government of Catalonia $\rightarrow$ Liberation of imprisoned independentistas $\rightarrow$ Extension of the desire for NO independence within Catalunya

5. From "Catalan Insolidarity with the rest of Spain" $\rightarrow$ Extension of the desire for independence in Catalonia $\rightarrow$ Independentist declarations of the Parliament of Catalonia $\rightarrow$ Acting of the Prosecutor's Office $\rightarrow$ Acting of the Courts of Justice $\rightarrow$ Non-compliance with laws or judicial resolutions in Catalonia $\rightarrow$ Charges police and imprisonment of independentistas $\rightarrow$ Extension of the desire for independence in Catalonia

These tours start from five of the six level III factors. There has not been added a sixth route from "Lack of proportionality of electoral systems in Spain" because from the other five already reached the significant factors that could be reached from the sixth.

That is, from the model it would be inferred that the eventual judicialization of the problem would imply, on the one hand, the improbability of Catalonia achieving independence, and on the other hand, the persistence of the desire for independence in a good part (approximately half) of its population, thus exacerbating the conflict, and that on the other hand the only viable solution to the conflict to which the methodology of the "Interpretive Structural Modeling" (ISM) used would be is a NEGOTIATION FROM THE SPANISH CONSTITUTION, either for it to continue demanding the territorial unity of Spain (article 2), either to modify said article in the opposite direction.

Thus, from the complex network of interactions between the different factors involved, it can be inferred that there are two possible ways of evolution of the conflict in Catalonia:

1.The police, judicial and penitentiary way, which would generate an intensification of the independence movement, although it would not foreseeably lead to the independence of Catalonia, due to the obstacles that it would encounter and the lack of external support.
2. The path of dialogue, which would generate in Catalonia an increase in the desire for permanence within Spain. Therefore, even in the hypothetical case that the dialogue led to a change in the Constitution that included the recognition of the right to self-determination, this would not lead to the independence of Catalonia, given the lack of majority support for it among its population .

Therefore, the real dilemma is not whether Catalonia becomes an independent State or not, which does not seem viable in any case, but between the conflict or the development of a harmonious relationship between Catalonia and the rest of Spain .

The path of dialogue could be implemented as follows:

1. The Catalan Parliament would formulate an Institutional Declaration promising in a solemn act to fulfill and enforce the Constitution in all its precepts with loyalty.

2. Before said solemn declaration, the central government proceeds to pardon all the imprisoned or fled politicians, allowing them to continue their normal political activity within the constitutional channels.

This political normalization would have two immediate consequences:

a) It would imply that all Catalan institutions normally participate in inter-regional institutions and that any claim can be raised and resolved in a consensual manner with the rest of the autonomous communities

b) Logically all the possibilities of changing the Constitution without any pre-established limit would be open. That is to say, the possible disjuncts Monarchy / Republic, Federal / Unitary State, Autonomic Financing by quotas / equal financing, etc., could be proposed as long as the initiatives are presented in the Spanish Cortes and submitted to a referendum of all citizens from Spain.

Thus we would have the following causal process expressed in the following annexed model:

\section{Constitutional recognition}

Institutional

\section{Political pardon}

normalization

Changes on the

Constitution

Our conclusion is, then, the following: unless we have forgotten to include in the model any very significant variable in the process, it does not seem that there are other possibilities of conflict resolution that are not a flexible negotiation, starting with the Constitution and eager to reach a lasting agreement. 


\section{CONSULTED BIBLIOGRAPHY}

Warfield, J.W. (1974), "Developing interconnected matrices i structural modelling", IEEE Transactions on Systems Men and Cybernetics, 4(1), pp. 51-81.

APSA (American Political Science Association), "La ciencia política y su incapacidad de predicción”, Jun 26, 2012. (www)

Attri R. Et al. "An ISM approach for modelling the enablers in the implementation of total Productive Maintenance (TPM), in International Journal System Assurance Engineering and Management, DOI: 10.1007, (2012

Attri, R.; Dev, N.; Sharma, V. (2013), "Interpretive Structural Modelling (ISM) approach: An Overview" Research Journal of Management Sciences, vol. 2(2), pp. 3-8.

Ballesteros, D.P.; Ballesteros, P.P.(2008), “Análisis estructural prospectivo aplicado al sistema logístico". Scientia et Technica, Año XIV nº 39, pp. 194-199.

Bolanos R., et al. "Using interpretive structural modelling in strategic decision making groups", Management Decision, 43 (6), 877-895 (2005)

Boscan Carrasquero, G. "La modelización formal en la ciencia política: usos, posibilidades y limitaciones,", Polit. Gob. vol 17, no. 1 México, Ene. 2010.

Bruce Bueno de Mesquita, "Can Game Theory Predict When Iran Will Get the Bomb?., NYT CLIVE THOMPSON, August 12, 2009

Centre d'Estudis d'Opinio (CEO). "Barómetro de Opinión Política de Junio 2017.

Dawkins, Richard, "El gen egoísta": las bases biológicas de nuestra conducta”, Salvat Ciencia, 2002.

Garson, G. David, "Social Science Computer Simulation: Its History, Design and Future" in Social Science Computer Review, vol, 12,pp.55-82 (1994)

Garson G. David, "Computerized Simulation in the Social Sciences: A Survey and Evaluation", Simulation and Gaming, en SAGE Journals on line (20 Agosto 2008)

Morton, Rebecca, "Methods and Models: A Guide to the Empirical Analysis of Formal MODELS in Political Science", N.Y. Cambridge Univ. Press., 2005.

Observer (The). "The Observer view on Catalan independence" (Observer editorial), 26/9/2018

Parra Luna, F. "Can Systems Theory predict the Evolution of Secessionism in Catalonia with respect to the Kingdom of Spain", Paper delivered at the 10th International Congress of the European Union for Systemics, Brussels, 15-17 October, 2018.

Rajesh Attri, Nikhil Dev., and Vivek Sharma, "Interpretive Structural Modelling (ISM) approach: An Overview", Research Journal of Management Sciences, Vol.2(23), 3-8 February 2013

Ravi V. And Shankar R., Analysis of interactions among the barriers of reverse logistics", Technological Forecasting and Social Change, 72, 1011-1029, 2005

Singh et al. "Modelling of critical sucesss factors for implementation of AMTYs, in Journal of Modelling in Management, 2(3), 232-250, (2007)

Stevens, J. "Political Scientists are Lousy Forecasters", New York Times (June 24, 2012)

Warfield J.A., Developing interconnected matrices in structural modelling, IEEE Transations on Systems Men and Cybernetics, 4(1), 51-81 (1974) 